\title{
The Impact of Age and Sex Concordance Between Patients and Physicians on Medication Adherence: A Population-Based Study
}

\author{
Shenzhen Yao (D) \\ Lisa Lix ${ }^{2}$ \\ Gary Teare ${ }^{3}$ \\ Charity Evans (D) \\ David Blackburn' \\ 'College of Pharmacy and Nutrition, \\ University of Saskatchewan, Saskatoon, \\ Saskatchewan, Canada; ${ }^{2}$ Department of \\ Community Health Sciences of Rady \\ Faculty of Health Sciences, University of \\ Manitoba, Winnipeg, Manitoba, Canada; \\ ${ }^{3}$ Program Program Knowledge, Evidence \\ and Innovation, Provincial Population and \\ Public Health, Alberta Health Services, \\ Calgary, Alberta, Canada
}

Correspondence: David Blackburn College of Pharmacy and Nutrition, University of Saskatchewan, 2A20.0I Health Sciences Building, 107 Wiggins Road, Saskatoon, Saskatchewan, S7N5E5, Canada

Tel + I 306966208 I

Fax +l 3069666377

Email d.blackburn@usask.ca
Purpose: Age or sex concordance (same sex or same age range) may also be associated with medication adherence but was not fully investigated. We aim to quantify the impact of age and sex concordance on optimal adherence to statin medications.

Patients and Methods: A retrospective cohort study was conducted using population-based health administrative data from Saskatchewan, Canada. Participants were individuals newly initiated on statin medications between January 1, 2012, and December 31, 2017. The outcome was optimal adherence (proportion of days covered $\geq 80 \%$ ) measured at one year after the first statin claim. The independent variables were sex and age concordance (age within five years) between patients and prescribers. The association between adherence outcome and sex/age concordance was analyzed by multivariable logistic regression models using generalized estimating equations controlled by a package of potential confounding factors.

Results: Among 51,874 new statin users, 20.6\% $(\mathrm{n}=10,710)$ were age concordant with prescriber. The vast majority of age concordance occurred in patients younger than 66 years $(88.6 \%, 9,486 / 10,710)$. Sex concordance was observed in $62.8 \%(\mathrm{n}=32,551)$ of patients and age-sex combined concordance in $13.2 \%(\mathrm{n}=6,856)$. Among patients younger than 66 years ( $\mathrm{n}=36,641 / 51,874,70.6 \%$ ), age concordance did not have a significant impact on optimal adherence [adjusted OR $(\mathrm{aOR})=1.02,95 \%$ CI 0.97 to 1.07 ]. Weak association between sex concordance ( $\mathrm{aOR}=1.05,95 \%$ CI 1.00 to 1.11$)$, and age-sex combined concordance $(\mathrm{aOR}=$ $1.05,95 \%$ CI 0.99 to 1.12 ) was observed.

Conclusion: Age and sex concordance were not statistically significant predictors of optimal statin adherence. However, a weak association was detected for sex concordance. Future studies should examine this factor in different health care settings.

Keywords: medication adherence, age concordance, sex concordance, statin, observational study, cohort

\section{Introduction}

Over decades of research on medication adherence, the impact of age and sex has been evaluated countless times without a clear and consistent signal. ${ }^{1}$ Mathes et al concluded that the effects of patient age on medication adherence were heterogeneous after reviewing 22 systematic reviews published between January 1990 and June 2018. ${ }^{2,3}$ Similarly, a consistent impact of sex on medication adherence has not been demonstrated. ${ }^{2,3}$

Although age and sex of patients appear to have weak influences on medication adherence, few studies have evaluated the extent to which they may influence 
medication adherence through interactions with other factors. $^{4}$ The existence of interactions between demographic characteristics may be important for medication adherence. For example, Schoenthaler et al found that African-American patients under care of white physicians (ie, discordance on ethnicity) had lower medication adherence compared to white patients under care of white physicians. ${ }^{5}$ Presumably, this "concordance" of race may have facilitated a more effective or trustful relationship between physicians and patients, improving medication adherence. ${ }^{5,6}$ Indeed, a strong physician-patient relationship has clearly been associated with high medication adherence as multiple studies have identified trust and communication between patients and physicians as important factors. ${ }^{1,7-12}$ Thornton et al found that patientphysician concordance on age and sex has positive effect on communication and satisfaction of care. ${ }^{4}$ However, despite the strong connection between communication and medication adherence, the impact of age and/or sex concordance on medication adherence to prescribed medications has not been examined.

Based on these findings, we hypothesized that age and/ or sex concordance may also be associated with medication adherence through the presumed mechanism of facilitating a more effective patient-physician relationship. The aims of this study were: 1) To describe the frequency of age and/or sex concordance between prescribers and patients initiating statin medications; 2) To determine if age concordance, sex concordance, or age-sex combined concordance is associated with the occurrence of optimal adherence within the first year since initiating statin therapy.

\section{Methods}

\section{Data Sources}

The study was conducted using administrative databases for Saskatchewan, Canada. These databases, linked by a common encrypted identification number for each patient, include the provincial health insurance registry file, the physician service claims file, the physician registry file, the hospital discharge abstract database, the emergency services file, and the prescription drug claims files. ${ }^{13}$ The provincial health insurance registry file contains birth month/year, sex, rural/urban residence, provincial health insurance coverage start and end dates, and dissemination area (smallest standard geographic area for census data defined by Statistics Canada) ${ }^{14}$ code of residence. The latter is used to assign area-level median household income based on 2006 census data. ${ }^{15}$ The physician service claims file contains the date of the service, setting (in-hospital or out-patient), diagnosis [using threedigit International Classification of Diseases (ICD-9) codes], ${ }^{16}$ physician identification number (encrypted), physician specialty, the billing code pertaining to the service provided, and the remuneration type of the provider [fee-for-service (FFS)/non-fee-for-service type (NFFS)]. Although non-fee-for-service physicians are encouraged to submit "shadow claims", compliance is not enforced, and not all claims are captured. However, the percentage of missing shadow claims is not likely to be large, given previous Canadian research. ${ }^{17}$ The physician registration file contains physicians' birth year, sex, and an indicator to distinguish general physicians (GPs) from other specialty physicians. It also provides information on country of medical training of physicians in Saskatchewan. The hospital discharge abstract database contains admission and discharge dates, up to 25 diagnostic codes ICD-9 (2001 and before) or ICD-10-CA (after 2001), ${ }^{16,18}$ and an indicator about the event type (ie, whether it was for acute or alternative care, of which a patient occupied a bed but did not require the intensity of services as for acute care). ${ }^{19}$ The emergency service file provides admission and discharge date of visits to emergency departments. The prescription drug claims files capture dispensation claims of prescription medications, each containing a Health Canada drug identification number (DIN), a dispensation date, the quantity dispensed, total cost (including medication acquisition cost and markup/dispensing fee), and the proportion covered by government insurance. The drug files only contain claims in outpatient settings.

\section{Study Design and Population}

We conducted a retrospective cohort study of new statin users at least 18 years of age who received their first statin medication between January 1, 2012, and December 31, 2017. New users were defined as receiving no dispensations for a statin medication in the previous five years. The date of the first dispensation of a statin medication was the index date, and the patients were followed for 365 days. For each patient, a single statin prescriber was identified using the following criteria: a) the physician with a GP specialty, and b) the GP identified on the highest number of statin dispensation claims for a specific patient (compared to all other GPs) during the 365 day follow-up 
period. In Saskatchewan, GPs provide primary care to the majority of patients with chronic conditions.

Exclusion criteria included: missing age or sex of patients or statin prescribers; unable to determine the remuneration type of statin prescribers; inability to follow patients between 1825 days before and 365 days after the first statin claim due to loss of beneficiary status (including death), or admission to a long term care facility; patients admitted to an out-of-province hospital in the year after initiating the statin; a diagnosis of pregnancy (ICD-9: 641676, V27; ICD-10 and ICD-10-CA: O1, O21-95, O98, O99, Z37) within one year before or after the index date; or if none of their statin prescribers was a GP physician.

\section{Patient and Public Involvement}

Patients and/or the public were not involved in the design or conduct of this study.

\section{Outcome Measures}

The primary outcome was optimal adherence to statin medications defined by the proportion of days covered (PDC) of at least $80 \%{ }^{20,21}$ PDC was measured over the 365 days following the index date using the sum of the number of pills dispensed divided by 365 (assuming once-daily satin dosing), deducting the number of days spent in a hospital for acute care if applicable. ${ }^{22}$ Pills dispensed during overlapping/early refills were counted in the numerator for the primary analysis and removed in sensitivity analyses. Switching between different statin medications was allowed. The PDC captures underutilization from either drug discontinuation or late refills due to missed doses.

\section{Age/Sex Concordance Between Patients and Their Statin Prescriber}

Age of each patient and their corresponding statin prescriber was determined on each patient's index date. Patients were categorized as age-concordant if their age was within five years above or below the prescriber's age; sexconcordance was assigned if the patient and the prescriber were of the same sex. Age-sex combined concordance was determined if both age and sex concordance were satisfied.

\section{Covariates}

Numerous patient and provider-related covariates were identified to minimize confounding based on previous studies. ${ }^{1}$ Covariates were measured during the 365 days prior to the index date if not otherwise specified. Patient- related covariates included patient characteristics [age, sex, and residence (rural/urban) on the index date]; socioeconomic status [income level based on census area-level median household income quintiles (lowest $=1$, highest $=5$ ) on the index date], ${ }^{23,24}$ treatment factors [number of distinct prescription medications (by the Anatomical Therapeutic Chemical Classification System) ${ }^{25}$ ]; healthcare system factors [percentage of prescription medication cost paid by government health insurance], and patient health/healthcare utilization factors [number of out-patient visits (to GPs and to specialists, respectively), number of hospitalizations for acute care, number of emergency department visits, Charlson comorbidity score, ${ }^{26}$ and presence of patient comorbidities (yes/no) used in published models of medication adherence]. ${ }^{27}$ The comorbidities included osteoporosis, rheumatoid arthritis, hypertension, stroke, ischemic heart disease, acute myocardial infarction, heart failure, multiple sclerosis, Parkinson's disease, Alzheimer's disease and dementia, epilepsy, asthma, chronic obstructive pulmonary disease, diabetes, mood and anxiety diseases, schizophrenia, and cancer. ${ }^{27}$ Comorbidities were identified using validated case definitions developed by the Canadian Chronic Disease Surveillance System and were based on diagnoses recorded in the service claims file, hospital discharge abstract database, and medications in the prescription drug claims dating back to January $1^{\text {st }}, 1996 .{ }^{27}$

Prescriber-related covariates included the statin prescribers' age and sex on the index date, country of medical graduation, as well as a categorical variable of remuneration type [fee-for-service (FFS) or non-fee-for-service" (NFFS)]. These covariates were adopted in previous studies on quality of care provided by physicians. ${ }^{28-30}$ The remuneration type for each prescriber was determined using physician-specific claims to all their patients (ie, not only limiting to study patients) in the physicianservice file between 365 days prior and 365 days on and after the index date. FFS remuneration practitioners were defined by at least $80 \%$ of claims coded as the FFS type; NFFS remuneration practitioners were defined by at least $80 \%$ of claims coded as the NFFS type. Over $95 \%$ of GP physicians could be categorized into one of the groups using this approach.

Finally, we included a variable identifying patients receiving comprehensive continuity of care where the patient's statin prescriber also: 1) claimed at least one comprehensive medical exam on the patient; and 2) had the highest number of service claims to the study patient compared to all other GPs. We found that this measure of 
continuity of care is superior to traditional approaches (unpublished). The comprehensive continuity of care was estimated during the 365 days after the index date (inclusive).

\section{Statistical Analysis}

The frequency of age and sex concordance was described using percentages, medians and the interquartile range (IQR), as appropriate. Since age concordance was strongly influenced by patient age, we stratified the cohort based on age ( $>65$ years, or $\leq 65$ years, respectively).

For each of the concordance variables under analysis (age concordance, sex concordance, and age-sex combined concordance), we fit univariate logistic regression models using optimal adherence (PDC $\geq 80 \%$ ) as the dependent variable. Generalized estimating equations (GEE) were used to account for the clustering of patients within prescribers in all univariate and multivariable models. ${ }^{31}$ GEE models using different types of working covariance matrices were compared and the one with the smallest quasi-Akaike's information criterion (QAIC) statistic was selected as the final model. Odds ratios (ORs) and 95\% confidence intervals $(95 \% \mathrm{CI})$ for the concordance variables were obtained from the robust estimators.

Next, we estimated adjusted effects of the concordance variables using multivariable logistic regression models that included patient and prescriber-related covariates. Multicollinearity between a concordance variable and each covariate was examined using the variance inflation factor (VIF) derived from a regression model. If the VIF value was greater than 2.5 , the covariate was removed. Two multivariable models were constructed; one included both of the age concordance and sex concordance variables, while the second included the age-sex combined concordance variable.

Age concordance with prescribers was uncommon for patients over 65 years of age; therefore, due to the potential bias associated with disproportionately high number of elderly patients in the discordant (vs concordant) age group, all adjusted models were tested in a subgroup of patients age at or below 65 years.

In sensitivity analyses, we changed age concordance to 10 years (ie, instead of five) and we modified the threshold of optimal adherence to PDC $\geq 70 \%$, and PDC $\geq 90 \%$. In addition, we examined the effect of sex concordance within stratified groups based on the statin prescribers' sex. For each prescriber sex group, we reported the proportion of patients achieving optimal adherence. We also repeated the analysis of sex concordance among the entire cohort of statin users (ie, not just those $\leq 65$ ).

SAS statistical software, version 9.4, (SAS Institute Inc., Cary, NC, USA) was used to conduct all analyses. ${ }^{32}$

\section{Ethical Considerations}

Ethics approval (certificate number: 14-143) was granted by the University of Saskatchewan Biomedical Research Ethics Board (REB). Data access was granted at the Saskatchewan Health Quality Council under data sharing agreements with the Saskatchewan Ministry of Health and eHealth Saskatchewan. The University of Saskatchewan REB approved to waive the informed consent from study participants for the following reasons: 1) It was a retrospective study using historical data dated back to 1996; 2) All participants were anonymized by encrypted IDs; 3) Privacy of individuals was further protected by suppressing results from any group of fewer than six participants.

\section{Results}

Between January 1, 2012, and December 31, 2017, 180,010 patients received statin medications. Among them, 21,149 were excluded for missing demographic information, age $<18$ on the index date, or lacking continuous beneficiary status. Of the 58,549 patients who were defined as new statin users, 3,405 (5.8\%) were excluded for admission to a long term care facility, staying in an out-of-province hospital, pregnancy/delivery, or having zero service claims by a GP within the follow-up period. Further, 3,270 (5.5\%) patients were excluded for having a statin prescriber with missing birth year, sex, or remuneration type. The final cohort was comprised of 51,874 new users of statin medications [Figure 1].

Initially 1,789 GPs had been identified as statin prescribers for at least one new statin user. Among them, 227 $(12.7 \%)$ prescribers were excluded for missing data on year of birth, sex, or undetermined remuneration type. Thus, 1,562 statin prescribers remained in the study, and were linked to at least one of the 51,874 patients [Figure 1].

The utility of using statin dispensation claims to identify a single GP prescriber was supported by the underlying data. There were 415,564 claims of statin medications for the cohort patients within the follow-up period. Most of these claims $(85.5 \%, 355,206 / 415,564)$ originated from GPs who were identified as statin prescribers, while only $14.5 \%(60,358 / 415,564)$ from other GPs or specialists. The median number of total statin 


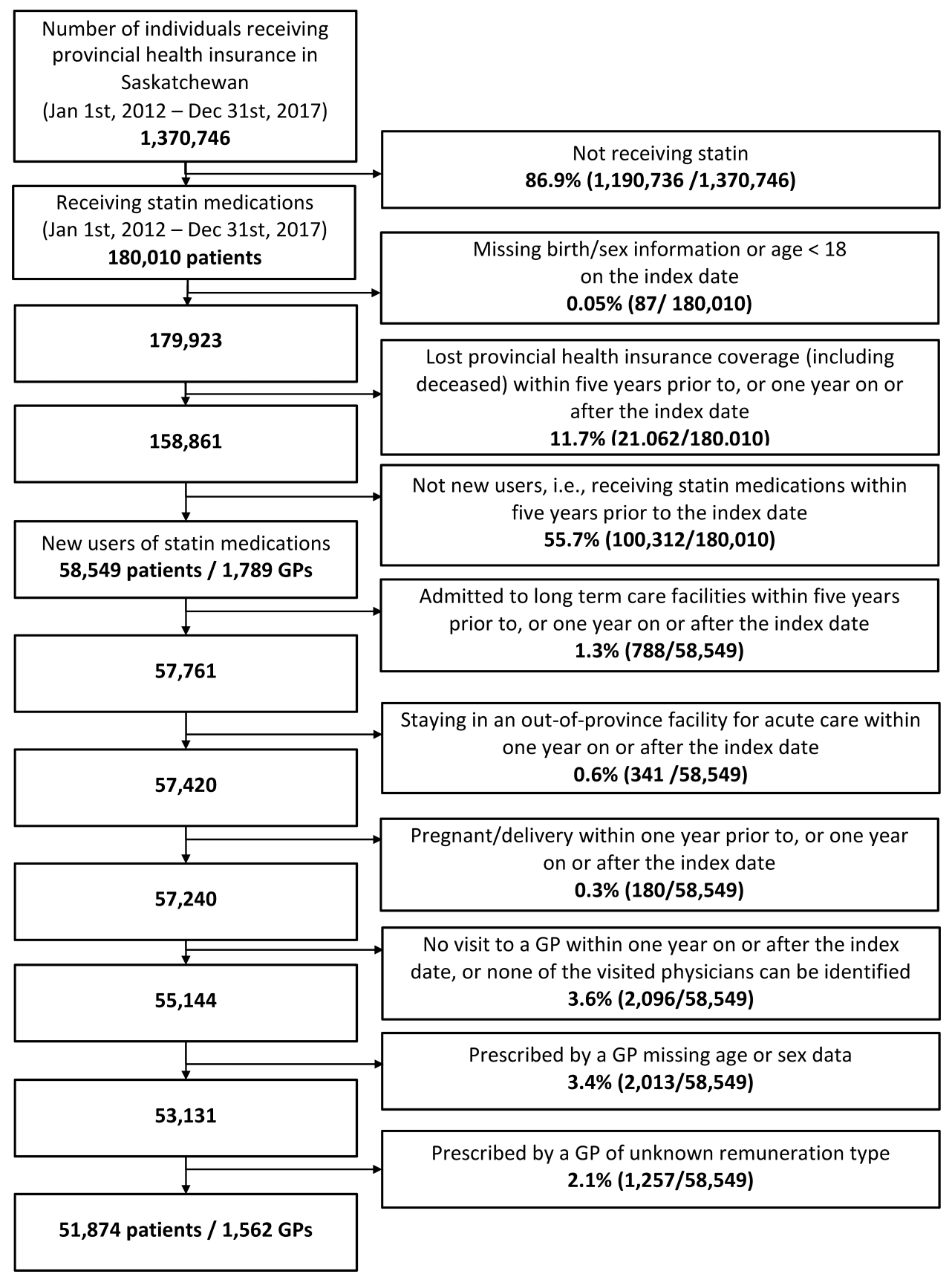

Figure I Patient flow.

Notes: Index date $=$ the first date receiving a statin medication between January $1^{\text {st }}, 2012$ and December $3 I^{\text {st }}, 2017$.

Abbreviation: GP, general practioner.

claims per patient during the follow-up period was 9.0 (IQR, 4.0/11.0) and the median number of statin claims from the identified prescriber (ie, the most frequently listed GP) was 7.0 (IQR, 3.0/10.0). Other prescribers (eg, other GPs or specialists) accounted for only 2.0 statin claims per patient (IQR, 1.0/3.0). Moreover, of all patients in the cohort, 31,539/51,874 (60.8\%) received all statin prescriptions from a single GP [Table 1].
The median age of patients on the index date was 59.0 years (IQR, 51.0/67.0), and 43.9\% $(22,781 / 51,874)$ were females [Table 1]. Among the 51,874 patient-prescriber pairs, the median age of physicians on the index date was 50.0 (IQR, 40.0/59.0). There were 36.0\% (562/ 1,562) female statin prescribers and they appeared in 13,532 (26.1\%) patient-prescriber pairs [Table 1].

The median age of patients was 10 years older than the median age of prescribers (IQR, $-3 / 22$ years). Age differences 
Table I Baseline Characteristics of New Statin Users

\begin{tabular}{|c|c|c|c|}
\hline & Total & Age $\leq 65$ & Age $>65$ \\
\hline & $n=5 I, 874$ & $n=36,641$ & $n=15,233$ \\
\hline Age of patients, median (IQR) & $59.0(51.0,67.0)$ & $54.0(48.0,60.0)$ & $73.0(69.0,79.0)$ \\
\hline Age of prescribers ${ }^{\mathrm{a}}$, median (IQR) & $50.0(40.0,59.0)$ & $50.0(40.0,59.0)$ & $49.0(40.0,59.0)$ \\
\hline Age difference (patient minus prescriber), median (IQR) & $10.0(-3.0,22.0)$ & $4.0(-7.0,14.0)$ & $25.0(15.0,35.0)$ \\
\hline Age concordance ${ }^{\mathrm{b}}, \mathrm{n}(\%)$ & $10,710(20.6)$ & $9,486(25.9)$ & I,224 (8.0) \\
\hline \multicolumn{4}{|l|}{ Age discordance $^{c}$} \\
\hline Patients $>5$ years younger than prescribers, $n(\%)$ & $10,804(20.8)$ & $10,575(28.9)$ & $229(1.5)$ \\
\hline Patients $>5$ years older than prescribers, $\mathrm{n}(\%)$ & $30,360(58.5)$ & $16,580(45.2)$ & $13,780(90.5)$ \\
\hline Female patients, $\mathrm{n}(\%)$ & $22,78 \mid(43.9)$ & $|5,22|(4 \mid .5)$ & $7,560(49.6)$ \\
\hline Female prescribers/prescribers of all sex, $\mathrm{n}(\%)$ & $562 / 1,562(36.0)$ & $545 / 1,495(36.5)$ & $465 / 1,309(35.5)$ \\
\hline Patients whose statin prescribed by a female GP, n (\%) & $13,532(26.1)$ & $9,867(26.9)$ & $3,665(24.1)$ \\
\hline Sex concordance, n (\%) & $32,55 I(62.8)$ & $23,649(64.5)$ & $8,902(58.4)$ \\
\hline Age-sex combined concordance, $n$ (\%) & $6,856(13.2)$ & $6,133(16.7)$ & $723(4.7)$ \\
\hline Statin claims per patient, median (IQR) & $9.0(4.0,11.0)$ & $9.0(4.0,11.0)$ & $10.0(5.0,12.0)$ \\
\hline $\begin{array}{l}\text { Statin prescribers per patient, } \\
\text { median (IQR) }\end{array}$ & $1.0(1.0,2.0)$ & $1.0(1.0,2.0)$ & $1.0(1.0,2.0)$ \\
\hline Patients with a unique statin prescriber, $\mathrm{n}$ (\%) & $31,539(60.8)$ & $23,543(64.3)$ & $7,996(52.5)$ \\
\hline Patients with multiple statin prescribers, $\mathrm{n}(\%)$ & $20,335(39.2)$ & $13,098(35.7)$ & $7,237(47.5)$ \\
\hline $\begin{array}{l}\text { Statin claims by the paired prescriber, } \\
\text { median (IQR) }\end{array}$ & $7.0(3.0,10.0)$ & $7.0(3.0,10.0)$ & $7.0(4.0,11.0)$ \\
\hline \multirow[t]{3}{*}{ Statin claims by other prescribers, median (IQR) } & $2.0(1.0,3.0)$ & $2.0(1.0,3.0)$ & $2.0(1.0,3.0)$ \\
\hline & Total & age $\leq 65$ & Age $>65$ \\
\hline & $n=5 I, 874$ & $n=36,64 I$ & $n=15,233$ \\
\hline $\mathrm{I}+$ acute care hospitalizations ${ }^{\mathrm{d}}, \mathrm{n}(\%)$ & II,493 (22.2) & $6,154(16.8)$ & $5,339(35.0)$ \\
\hline GP visits ${ }^{\mathrm{d}}$, median (IQR) & $6.0(3.0,9.0)$ & $5.0(3.0,8.0)$ & $7.0(4.0,11.0)$ \\
\hline Specialist visits ${ }^{\mathrm{d}}$, median (IQR) & $2.0(0.0,6.0)$ & $2.0(0.0,5.0)$ & $4.0(1.0,10.0)$ \\
\hline I+ emergency department visits ${ }^{\mathrm{d}}, \mathrm{n}(\%)$ & $10,952(21.1)$ & $6789(18.5)$ & $4163(27.3)$ \\
\hline \multicolumn{4}{|l|}{ Income level $^{\mathrm{d}}$, n (\%) } \\
\hline I (lowest) & $9,569(18.4)$ & $6,772(18.5)$ & $2,797(18.4)$ \\
\hline 2 & $9,500(18.3)$ & $6,728(18.4)$ & $2,772(18.2)$ \\
\hline 3 & $9,540(18.4)$ & $6,718(18.3)$ & $2,822(18.5)$ \\
\hline 4 & $10,685(20.6)$ & $7,519(20.5)$ & $3,166(20.8)$ \\
\hline 5 (highest) & $9,782(18.9)$ & $6,857(18.7)$ & $2,925(19.2)$ \\
\hline missing & $2,798(5.4)$ & $2,047(5.6)$ & $751(4.9)$ \\
\hline Patients living in a rural area ${ }^{\mathrm{d}}, \mathrm{n}(\%)$ & $15,830(30.5)$ & $10,729(29.3)$ & $5,101(33.5)$ \\
\hline Charlson comorbidity score ${ }^{d}>0, n(\%)$ & $16,988(32.7)$ & $9,463(25.8)$ & $7,525(49.4)$ \\
\hline
\end{tabular}

Notes: ${ }^{a}$ Age of individual prescribers was re-calculated for each of their patients on the date of the earliest statin dispensation; ${ }^{b}$ Age concordance was defined as patient age falling within five years above or below the prescriber's age; 'Age discordance was defined as patient age falling at least five years above or below the prescriber's age; ${ }^{d}$ Characteristics measured within 365 days prior to the date of the earliest statin dispensation.

Abbreviations: IQR, interquartile range; GP, general practitioners.

were higher among patients over 65 (median difference $=25$ years, IQR: $15 / 35$ years) versus those $\leq 65$ years (median difference $=4$ years, IQR: $-7 / 14$ years) $[$ Table 1]. Overall, only $20.6 \%(n=10,710)$ of the entire cohort were concordant by age (ie, within 5 years) with their statin prescriber on the index date. Most of these age concordant patients were 65 years or younger $(88.6 \%$ or $9,486 / 10,710)$. Among those older than 65 , only $8.0 \%(1,224 / 15,233)$ were age concordant to their statin prescribers and $90.5 \%(13,780 / 15,233)$ were more than five years older [Table 1]. Sex concordance was observed in $62.8 \%(32,551 / 51,874)$ of patients. Age-sex combined concordance was relatively infrequent, observed in only $13.2 \%$ $(6,856 / 51,874)$ of patients overall and $16.7 \%(6,133 / 36,641)$ of patients $\leq 65$ years. Among those older than 65 , there were only $4.7 \%(723 / 15,233)$ patients were both age and sex concordant [Table 1]. 
In the multivariate models, the GEE method using the exchangeable working covariance matrix had the smallest QAIC value among the tested structures. Logistic regression analysis did not detect an impact of age concordance on optimal adherence [unadjusted OR (uOR) = $1.02,95 \%$ CI 0.96 to 1.08 ; adjusted OR $(\mathrm{aOR})=1.02$, $95 \%$ CI 0.97 to 1.07 , Table 2]. The odds ratios of sex concordance on optimal adherence were $\mathrm{uOR}=1.05$, (95\% CI 1.01 to 1.10$)$, and $\mathrm{aOR}=1.05$ (95\% CI 1.00 to 1.11 ), while the impact of age-sex combined concordance was similar to sex concordance only: uOR $=1.06(95 \%$ CI 0.99 to 1.13$), \mathrm{aOR}=1.05(95 \%$ CI 0.99 to 1.12$)$ [Table 2]. Results were similar when analyses were repeated with age concordance measured by a broader range of years (ie, age \pm 10 years) or when changing the optimal adherence threshold to PDC $\geq 70 \%$, and PDC $\geq 90 \%$ (data not shown).

In stratified analyses of patients $\leq 65$ years of age, sex concordance was weakly associated with optimal adherence for patients with male prescribers (aOR $=1.06,95 \%$ CI 1.00 to 1.11, Table 2). Optimal adherence was observed in $50.9 \%(8961 / 17,601)$ of male patients with male prescribers versus $49.4 \%$ (4529/9173) of female patients with male prescribers [Table 3]. When restricting to patients of female prescribers the result was similar $(\mathrm{aOR}=1.05$, 95\% CI 0.95 to 1.16 ). In this subgroup, optimal adherence was observed in 49.8\% (3011/6048) of female patients under female prescribers versus $48.3 \%$ (1824/3779) of male patients under female prescribers [Table 3].

Within the subgroup of patients $>65$ years, sex concordance with male prescribers was significantly associated with optimal adherence $(\mathrm{aOR}=1.10,95 \%$ CI 1.01 to 1.19) [Table 2]. Optimal adherence was observed in $63.9 \%(4,127 / 6,455)$ of male patients with male prescribers versus $60.6 \%(3,098 / 5,113)$ of female patients with male prescribers [Table 3]. For patients with a female prescriber, sex concordance was not associated with optimal adherence $(\mathrm{aOR}=0.96,95 \%$ CI 0.81 to 1.13$)$ [Table 2]. In this group, $60.3 \%(1,476 / 2,447)$ of female patients under female prescribers achieved optimal adherence versus $62.5 \%(761 / 1,218)$ of male patients under female prescribers [Table 3].

When the analysis of sex concordance was repeated among the entire cohort of statin users (ie, not just those $\leq 65$ years), results were consistent $(\mathrm{aOR}=1.05,95 \% \mathrm{CI}$ 1.00 to 1.10). Sex concordance among the subgroup of

Table 2 Odds Ratios (95\% Confidence Intervals) for Age and Sex Concordance with Optimal Adherence ${ }^{\mathrm{a}}$ Among New Statin Users

\begin{tabular}{|c|c|c|}
\hline & $\begin{array}{l}\text { Unadjusted Odds Ratio } \\
\text { (95\% Confidence Interval) }\end{array}$ & $\begin{array}{l}\text { Adjusted Odds Ratio } \\
\text { (95\% Confidence Interval) }\end{array}$ \\
\hline \multicolumn{3}{|l|}{ Patients aged $\leq 65$ years } \\
\hline Age concordance ${ }^{\mathrm{b}}$ (yes vs no) & $1.02(0.96,1.08)$ & $1.02(0.97,1.07)$ \\
\hline Sex concordance (yes vs no) & $1.05(1.01,1.10)^{d}$ & $1.05(1.00,1.11)$ \\
\hline Patients paired to a male prescriber & $1.02(0.97,1.07)$ & $1.06(1.00,1.11)$ \\
\hline Patients paired to a female prescriber & $1.15(1.05,1.27)$ & $1.05(0.95,1.16)$ \\
\hline Age-sex combined concordance & $1.06(0.99,1.13)$ & $1.05(0.99,1.12)$ \\
\hline \multicolumn{3}{|l|}{ Patients aged $>65$ years } \\
\hline Sex concordance (yes vs no) & $1.08(1.00,1.15)$ & $1.03(0.94,1.12)$ \\
\hline Patients paired to a male prescriber & $1.12(1.04,1.21)$ & $1.10(1.01,1.19)$ \\
\hline Patients paired to a female prescriber & $0.93(0.79,1.09)$ & $0.96(0.81,1.13)$ \\
\hline \multicolumn{3}{|l|}{ Patient age $\leq 65$ and $>65$} \\
\hline Sex concordance (yes vs no) & $1.03(0.99,1.07)$ & $1.05(1.00,1.10)$ \\
\hline Patients paired to a male prescriber & $1.00(0.96,1.05)$ & $1.06(1.02,1.11)$ \\
\hline Patients paired to a female prescriber & $1.12(1.02,1.22)$ & $1.03(0.94,1.13)$ \\
\hline
\end{tabular}

Notes: a Optimal adherence was defined as greater or equal to $80 \%$ measured by the proportion of days covered (PDC); ${ }^{\mathrm{b}} \mathrm{Age}$ concordance $=$ prescriber and patient age difference within \pm 5 years (age measured on the patient's date receiving the first dispensation of a statin medication); ${ }^{~} A d j u s t e d$ odds ratios were from models with covariates including: age, sex, residence (rural/urban),income quintile based on census area, number of distinct prescription medications, number of out-patient visits (to GPs and to specialists, respectively), percentage of prescription medication cost paid by government health insurance, number of hospitalizations for acute care, number of emergency department visits, Charlson comorbidity score, comorbidities (including osteoporosis, rheumatoid arthritis, hypertension, stroke, ischemic heart disease, acute myocardial infarction, heart failure, multiple sclerosis, Parkinson's disease, Alzheimer's disease and dementia, epilepsy, asthma, chronic obstructive pulmonary disease, diabetes, mood and anxiety diseases, schizophrenia, and cancer), status of comprehensive continuity of care, prescribers' age, sex, country of medical graduation, and remuneration type; ${ }^{d}$ Odds ratios are in bold if statistically significant using $95 \%$ confidence interval. 
Table 3 Frequency (\%) of Adherent Patients ${ }^{\mathrm{a}}$ by Sex Concordance Status

\begin{tabular}{|c|c|c|}
\hline & $\begin{array}{c}\text { Adherent Patients } \\
\text { in the Sex Concordance }{ }^{c} \text { Group } \\
\% \text { (Adherent Patients/Total in the Group) }\end{array}$ & $\begin{array}{c}\text { Adherent Patients } \\
\text { in the Sex Discordance }{ }^{\mathrm{d}} \text { Group } \\
\% \text { (Adherent Patients/Total in the Group) }\end{array}$ \\
\hline \multicolumn{3}{|l|}{ Patient age ${ }^{\mathrm{b}} \leq 65$ years } \\
\hline Paired to a male prescriber & $50.9(8,96 I / \mid 7,60 I)$ & $49.4(4,529 / 9,173)$ \\
\hline Paired to a female prescriber & $49.8(3,011 / 6,048)$ & $48.3(1,824 / 3,779)$ \\
\hline \multicolumn{3}{|l|}{ Patient age $>65$ years } \\
\hline Paired to a male prescriber & $63.9(4,127 / 6,455)$ & $60.6(3,098 / 5,113)$ \\
\hline Paired to a female prescriber & $60.3(1,476 / 2,447)$ & $62.5(76 \mid / 1,218)$ \\
\hline \multicolumn{3}{|l|}{ Patients of all age groups } \\
\hline Paired to a male prescriber & $54.4(13,088 / 24,056)$ & $53.4(7,627 / 14,286)$ \\
\hline Paired to a female prescriber & $52.8(4,487 / 8,495)$ & $51.7(2,585 / 4,997)$ \\
\hline
\end{tabular}

Notes: ${ }^{a}$ Adherent patients = patients achieving optimal adherence, ie, having a proportion of days covered by statin $\geq 80 \%$; ${ }^{b}$ Patient age was measured on the index date (the date receiving the first statin medication); ${ }^{\mathrm{c}} \mathrm{Sex}$ concordance $=$ the patient and the prescriber were of the same sex (eg, a female patient matching to a female prescriber); ${ }^{\mathrm{d} S e x}$ discordance $=$ the patient and the prescriber were of the different sex (eg, a female patient matching to a male prescriber).

patients with male physician prescribers was significantly associated with the odds of optimal adherence $(\mathrm{aOR}=1.06,95 \%$ CI 1.02 to 1.11$)$; however, sex concordance was not significantly associated with optimal adherence among patients with female physicians $(\mathrm{aOR}=1.03,95 \%$ CI 0.94 to 1.13$)$ [Table 2].

\section{Discussion}

We performed a population-based study of new statin users and their prescribing GPs to test whether age and/ or sex concordance influences the odds of optimal adherence. Age concordance was relatively infrequent, owing largely to the high percentage of patients who were over the age of 65 years. Although patients under the age of 65 were much more likely to be of similar age with their prescribing physician, no influence of age concordance on the odds of optimal adherence could be detected. In contrast, sex concordance between patients and physicians was observed more frequently $(62.8 \%$ in all age groups) and was weakly associated with optimal medication adherence, albeit with a small effect size. Although we cannot rule out the possibility that sex concordance with the prescribing physician may influence adherence of some patients, the impact appears to be small in our cohort. To our knowledge, our study is the first to investigate age and sex concordance on medication adherence, using population-based administrative data, and controlled by a wide range of patient- and physician-related covariates.

The literature suggests that patient-physician concordance on certain demographic characteristics may influence medication adherence. It appears that patients exhibiting the same race/ethnicity as their physician may be more likely to exhibit optimal adherence, presumably because of a more effective relationship and/or increased trust. Schoenthaler et al reported that the odds of high adherence among white patients treated by white physicians were $27 \%$ (OR: $1.27,95 \%$ CI 1.01 to 1.61 ) higher compared with patients receiving care from physicians with different ethnicity. ${ }^{5}$ Traylor et al found that Spanish speaking patients treated by Spanish speaking physicians were more likely to be adherent to medications compared to Spanish speaking patients treated by Non-Spanish speaking physicians $(50.6 \%$ vs $44.8 \%$, p < 0.05$) .^{6}$

We found a weak association between sex concordance and optimal adherence $(\mathrm{aOR}=1.05,95 \%$ CI 1.00 to 1.11$)$ in the initial analysis of patients $\leq 65$ years and a consistent finding was produced using the entire cohort of statin users (aOR 1.05, 95\% CI 1.00 to 1.10). Although the absolute impact of sex concordance appeared relatively small based on the descriptive analysis of these findings, it should be noted that these trends occurred in a health care system where patients are free to choose their GP physician. In other words, patients with strong preferences for a samesex prescriber would have likely been disproportionately represented in the sex-concordant group, especially in areas where multiple physicians were accessible to patients. Saskatchewan offers a universal health care system where patients are free to choose any general practitioner who is accepting new patients. Thus, the weak association observed in this analysis occurred despite a clear bias towards the null effect. 
Our study was not without limitations. First, the administrative data used in this study only captures dispensations but not consumption of medications. However, administrative data have been widely used in medication adherence research and have high validity. ${ }^{33}$ Second, administrative databases do not capture clinical data such as disease status, treatment effectiveness, or medication tolerability. Although misclassification of non-adherence could occur, it is unlikely to correlate with concordance groupings. Third, lack of randomization increases the chance of unmeasured confounding between concordance groups. Fourth, the income quintile data from the administrative database was old (from the census data of 2006). More recent data should be used when it is available in future studies. Fifth, patients above 65 years of age were excluded from the analyses of age concordance. However, the issue of age-concordance with physicians is not relevant to elderly patients as very few physicians in our cohort practiced during their elderly years (ie, median age of prescribers was 50 years). Finally, the impact of concordance was examined in a health care system that allows patients to choose their own providers. As discussed above, the direction of the bias is likely towards the null.

\section{Conclusion}

Age concordance between patients and statin prescribers does not appear to impact the odds of optimal adherence. However, a weak association was detected for a possible effect of sex concordance. Future studies should reexamine the impact of sex-concordance in areas where provider access is limited or in health systems that limit choice of providers. Sex-concordance may play a more important role in these contexts.

\section{Acknowledgments}

The authors acknowledge the Health Quality Council for use of de-identified data provided by the Saskatchewan Ministry of Health and eHealth Saskatchewan. The interpretation and conclusions contained herein do not necessarily represent those of the Government of Saskatchewan, the Saskatchewan Ministry of Health, or eHealth Saskatchewan.

\section{Disclosure}

All authors have completed the ICMJE uniform disclosure form at www.icmje.org/coi_disclosure.pdf and declare: David Blackburn is the Chair in Patient Adherence to
Drug Therapy within the College of Pharmacy and Nutrition, University of Saskatchewan. This position was created through unrestricted financial support from AstraZeneca Canada, Merck Canada, Pfizer Canada, and the Province of Saskatchewan's Ministry of Health. None of the sponsors were involved in developing this study or writing the manuscript. Shenzhen Yao, Lisa Lix, Gary Teare, and Charity Evans declare no conflicts of interest in this work.

\section{References}

1. De Geest S, Sabate E. Adherence to Long-Term Therapies: Evidence for Action. Geneva, Switzerland: World Health Organization (WHO); 2003.

2. Mathes T, Jaschinski T, Pieper D. Adherence influencing factors a systematic review of systematic reviews. Arch Public Health. 2014;72(1):37. PMID:25671110. doi:10.1186/2049-3258-72-37

3. Gast A, Mathes T. Medication adherence influencing factors-an (updated) overview of systematic reviews. Syst Rev. 2019;8(1):112. PMID:31077247. doi:10.1186/s13643-019-1014-8

4. Thornton RL, Powe NR, Roter D, Cooper LA. Patient-physician social concordance, medical visit communication and patients' perceptions of health care quality. Patient Educ Couns. 2011;85(3): e201-208. PMID:21840150. doi:10.1016/j.pec.2011.07.015

5. Schoenthaler A, Montague E, Baier Manwell L, Brown R, Schwartz MD, Linzer M. Patient-physician racial/ethnic concordance and blood pressure control: the role of trust and medication adherence. Ethn Health. 2014;19(5):565-578. PMID:24266617. doi:10.1080/13557858.2013.857764

6. Traylor AH, Schmittdiel JA, Uratsu CS, Mangione CM, Subramanian U. Adherence to cardiovascular disease medications: does patient-provider race/ethnicity and language concordance matter? J Gen Intern Med. 2010;25(11):1172-1177. PMID:20571929. doi:10.1007/s11606-010-1424-8

7. Leventhal MJ, Riegel B, Carlson B, De Geest S. Negotiating compliance in heart failure: remaining issues and questions. Eur $J \quad$ Cardiovasc Nurs. 2005;4(4):298-307. PMID:15893959. doi:10.1016/j.ejcnurse.2005.04.001

8. Bauer AM, Parker MM, Schillinger D, et al. Associations between antidepressant adherence and shared decision-making, patient-provider trust, and communication among adults with diabetes: diabetes study of Northern California (DISTANCE). J Gen Intern Med. 2014;29(8):1139-1147. PMID:24706097. doi:10.1007/ s11606-014-2845-6

9. Nguyen GC, LaVeist TA, Harris ML, Datta LW, Bayless TM, Brant SR. Patient trust-in-physician and race are predictors of adherence to medical management in inflammatory bowel disease. Inflamm Bowel Dis. 2009;15(8):1233-1239. PMID:19177509. doi:10.1002/ ibd.20883

10. Tran DQ, Barry V, Antun A, Ribeiro M, Stein S, Kempton CL. Physician trust and depression influence adherence to factor replacement: a single-centre cross-sectional study. Haemophilia. 2017;23 (1):98-104. PMID:27686244. doi:10.1111/hae.13078

11. Jaeger S, Weisshaupt S, Flammer E, Steinert T. Control beliefs, therapeutic relationship, and adherence in schizophrenia outpatients: a cross-sectional study. Am J Health Behav. 2014;38(6):914-923. PMID:25207516. doi:10.5993/AJHB.38.6.13

12. Blackstock OJ, Addison DN, Brennan JS, Alao OA. Trust in primary care providers and antiretroviral adherence in an urban HIV clinic. $J$ Health Care Poor Underserved. 2012;23(1):88-98. PMID:22643464. doi:10.1353/hpu.2012.0006 
13. Saskatchewan Ministry of Health Data Warehouse Overview (Internal Document). 2019.

14. Statistics Canada. Dissemination area: detailed definition. Available from: https://www150.statcan.gc.ca/n1/pub/92-195-x/2011001/geo/ da-ad/def-eng.htm. Accessed: January 1, 2020.

15. Statistics Canada. 2006 Census Data, Income and Earnings. Ottowa: Statistics Canada; 2006. Available from https:/www12.statcan.gc.ca/ census-recensement/2006/dp-pd/tbt/St-eng.cfm? LANG= E\&Temporal $=2006 \&$ APATH $=3 \&$ THEME $=81 \&$ FREE $=0 \& G R P=1$. Accessed April 19, 2019.

16. WHO. International Classification of Diseases: [9th] Ninth Revision, Basic Tabulation List with Alphabetic Index. Geneva, Switzerland: World Health Organization (WHO); 1978.

17. Lix LM, Kuwornu JP, Kroeker K, et al. Estimating the completeness of physician billing claims for diabetes case ascertainment using population-based prescription drug data. Health Promot Chronic Dis Prev Can. 2016;36(3):54-60. PMID:26959724. doi:10.24095/ hpcdp.36.3.02

18. CIHI. Canadian Coding Standards for Version 2018 ICD-10-CA and CCI. Ottawa, ON: Canadian Institute for Health Information (CIHI); 2018.

19. CIHI. Definitions and Guidelines to Support ALC Designation in Acute Inpatient Care. Ottawa, ON: Canadian Institute for Health Information (CIHI); JunE 30, 2019. Available from: https://www. cihi.ca/sites/default/files/document/acuteinpatientalcdefinitionsandguidelines_en.pdf. Accessed December 29, 2021.

20. Stolpe S, Kroes MA, Webb N, Wisniewski T. A systematic review of insulin adherence measures in patients with diabetes. J Manag Care Spec Pharm. 2016;22(11):1224-1246. PMID:27783551. doi:10.18553/jmcp.2016.22.11.1224

21. Raebel MA, Schmittdiel J, Karter AJ, Konieczny JL, Steiner JF. Standardizing terminology and definitions of medication adherence and persistence in research employing electronic databases. Med Care. 2013;51(8Suppl 3):S11-21. PMID:23774515. doi:10.1097/ MLR.0b013e31829b1d2a

22. Bartlett LE, Pratt N, Roughead EE. Does tablet formulation alone improve adherence and persistence: a comparison of ezetimibe fixed dose combination versus ezetimibe separate pill combination? $\mathrm{Br}$ $J \quad$ Clin Pharmacol. 2017;83(1):202-210. PMID:27517705. doi: $10.1111 /$ bcp. 13088
23. Alsabbagh MW, Lemstra M, Eurich D, et al. Socioeconomic status and nonadherence to antihypertensive drugs: a systematic review and meta-analysis. Value Health. 2014;17(2):288-296. PMID:24636389. doi:10.1016/j.jval.2013.11.011

24. Alsabbagh MW, Lix LM, Eurich D, Wilson TW, Blackburn DF. Multiple-domain versus single-domain measurements of Socioeconomic Status (SES) for predicting nonadherence to statin medications: an observational population-based cohort study. Med Care. 2016;54(2):195-204. PMID:26683784. doi:10.1097/MLR.0000 000000000468

25. WHO. The anatomical therapeutic chemical classification system; 2003. Available from: https://www.whocc.no/atc/structure_and_prin ciples/. Accessed February 1, 2020.

26. Quan H, Sundararajan V, Halfon P, et al. Coding algorithms for defining comorbidities in ICD-9-CM and ICD-10 administrative data. Med Care. 2005;43(11):1130-1139. PMID:16224307. doi:10.1097/01.mlr.0000182534.19832.83

27. Government of Canada. Chronic Diseases Defined by Canadian Chronic Disease Surveillance System (CCDSS). Ottowa, Canada: Government of Canada. Available from: https://www.canada.ca/en/public-health/ser vices/chronic-diseases.html. Accessed September 20, 2019.

28. Watson DE, Katz A, Reid RJ, Bogdanovic B, Roos N, Heppner P. Family physician workloads and access to care in Winnipeg: 1991 to 2001. CMAJ. 2004;171(4):339-342. PMID:15313992. doi:10.1503/ cmaj.1031047

29. Reid RO, Friedberg MW, Adams JL, McGlynn EA, Mehrotra A. Associations between physician characteristics and quality of care. Arch Intern Med. 2010;170(16):1442-1449. PMID:20837830. doi:10.1001/archinternmed.2010.307

30. Tsugawa Y, Jena AB, Orav EJ, Jha AK. Quality of care delivered by general internists in US hospitals who graduated from foreign versus US medical schools: observational study. BMJ. 2017;356:j273. PMID:28153977. doi:10.1136/bmj.j273

31. Zeger SL, Liang KY, Albert PS. Models for longitudinal data: a generalized estimating equation approach. Biometrics. 1988;44 (4):1049-1060. PMID:3233245. doi:10.2307/2531734

32. SAS Institute INC. SAS for Windows Version 9.4. Cary, NC, USA; 2011.

33. Strom BL, Kimmel SE. Textbook of Pharmacoepidemiology. John Wiley; 2013.
Patient Preference and Adherence

\section{Publish your work in this journal}

Patient Preference and Adherence is an international, peer-reviewed, open access journal that focusing on the growing importance of patient preference and adherence throughout the therapeutic continuum. Patient satisfaction, acceptability, quality of life, compliance, persistence and their role in developing new therapeutic modalities and compounds to optimize clinical outcomes for existing disease states are major areas of interest for the journal. This journal has been accepted for indexing on PubMed Central. The manuscript management system is completely online and includes a very quick and fair peer-review system, which is all easy to use. Visit http:// www.dovepress.com/testimonials.php to read real quotes from published authors. 